\title{
p53 and Gli combine forces
}

Enchondromas are benign cartilage tumours found in the metaphyses of bones (the location of growth plates) that can progress to malignant chondrosarcoma. Benjamin Alman and colleagues previously created a

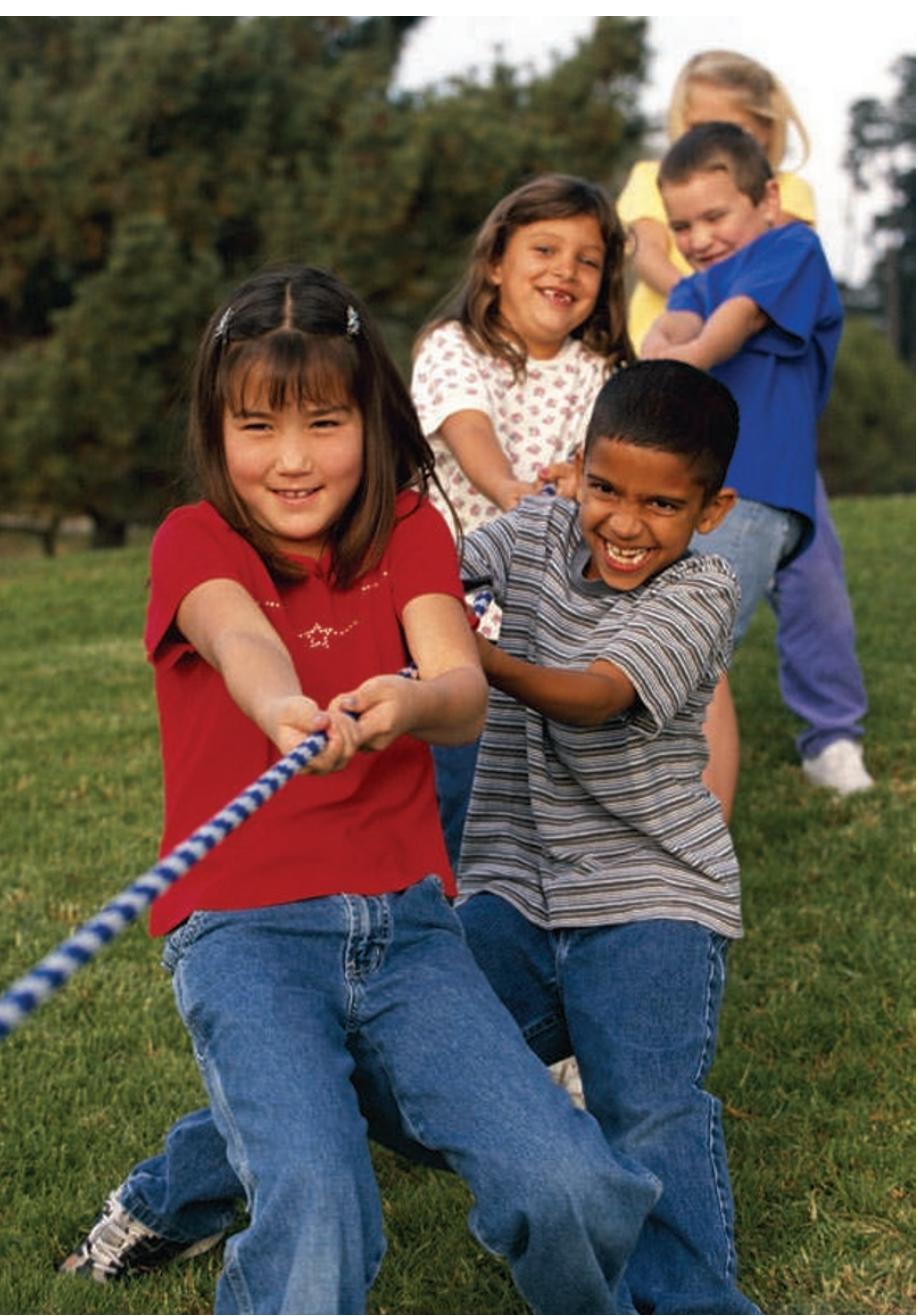

BRAND X mouse model of enchondroma in which Gli2 is expressed in chondrocytes under the control of the type

II collagen ( $\underline{\text { Col2a1 }}$ ) promoter (Col2a1-Gli2 mice); this is similar to the human disease, which has constitutive activation of transcription by the Gli family of transcription factors. As TP53 is mutated or abnormal in around one-third of chondrosarcomas, Alman and colleagues examined the role of p53 loss in enchondroma progression.

Col2a1-Gli2 mice were crossed with $\operatorname{Trp53} 3^{+-}$mice (Col2a1-Gli2; $\operatorname{Trp53^{+/-}}$ mice), and the authors showed that these mice had larger cartilage lesions, similar in appearance to low-grade chondrosarcomas, with more cellularity and pleiomorphic nuclei than Col2a1-Gli2; $\operatorname{Tr} p 53^{+/+}$ mice. Higher-grade sarcomas developed in $6 \%$ of the mice and these sarcomas had Trp53 loss of heterozygosity.

How do GLI2 and loss of p53 cooperate to induce chondrosarcomas? Collagen type X (COL10A1), a marker of differentiation, was higher in the fetal growth plates of Col2a1-Gli2; Trp53-/- mice than in wild-type mice. The authors found that the region of differentiated cells expressing COL10A1 increased in length as a result of decreased apoptosis in mice expressing GLI2 and lacking p53, compared with either GLI2 expression or p53 loss alone, indicating that GLI2 and p53 can negatively and positively regulate apoptosis, respectively.
Using gene expression profiling, the authors identified insulin-like growth factor binding protein 3 (IGFBP3), a known p53 target, as a candidate for mediating the effects of GLI2 and p53 on apoptosis. Quantitative PCR showed that IGFBP3 expression was lower in human enchondromas than in normal growth plates and even lower in chondrosarcomas (and therefore could be a biomarker of tumour grade or prognosis). Chromatin immunoprecipitation confirmed the presence of a GLI2-binding site in the Igfbp 3 promoter, and Igfbp 3 expression was additively decreased by GLI2 expression and p53 loss. Several lines of evidence showed that IGFBP3, probably through the sequestration of IGF and inhibition of IGF receptor signalling, could induce apoptosis and reduce COL10A1 expression in growth plate chondrocytes from Col2a1-Gli2; Trp53 ${ }^{-/-}$mice. Finally, Col2a1-Gli2 mice lacking one Igf2 allele had significantly fewer cartilage lesions than Col2a1-Gli2;Igf2 $2^{+/+}$ mice, suggesting that the inhibition of IGF signalling could be a possible therapeutic for chondrosarcoma, for which there is currently no universally effective chemotherapy.

Sarah Seton-Rogers

ORIGINAL RESEARCH PAPER Ho, L. et al. Gli2 and $\mathrm{p} 53$ cooperate to regulate IGFBP-3-mediated chondrocyte apoptosis in the progression from benign to malignant cartilage tumors. Cancer Cell 16, 126-136 (2009) 